\title{
Möbius strip microlasers
}

\author{
Stefan Bittner ${ }^{1}$, Yalei Song ${ }^{2,3}$, Yann Monceaux ${ }^{2}$, Kimhong Chao $^{2}$ and Héctor M. Reynoso de la Cruz $^{2,4}$, \\ Clément Lafargue $^{2}$, Dominique Decanini ${ }^{5}$, Barbara Dietz ${ }^{3}$, Joseph Zyss ${ }^{2}$, Alain Grigis ${ }^{6}$, \\ Xavier Checoury ${ }^{5}$, Mélanie Lebental ${ }^{2}$ \\ 1. Chair in Photonics, LMOPS EA-4423 Laboratory, CentraleSupélec and Université de Lorraine, 2 rue Edouard Belin, Metz 57070, France \\ 2. Laboratoire Lumière, Matière et Interfaces (LuMIn), CNRS, ENS Paris-Saclay, CentraleSupélec, 91190 Gif-sur-Yvette, France \\ 3. School of Physical Science and Technology, and Key Laboratory for Magnetism and Magnetic Materials of MOE, Lanzhou University, \\ Lanzhou, Gansu 730000, China \\ 4. Science and Engineering Division of the University of Guanajuato, León, Gto., Mexico \\ 5. Centre de Nanosciences et de Nanotechnologies, CNRS, Université Paris-Saclay, 10 Boulevard Thomas Gobert, Palaiseau, 91120, France \\ 6. Laboratoire d'Analyse, Géométrie et Applications, CNRS UMR 7539, Université Sorbonne Paris Cité, Université Paris 13, Institut Galilée, \\ 99 avenue Jean-Baptiste Clément, 93430 Villetaneuse, France
}

Direct laser writing [1] enables the fabrication of almost arbitrary three-dimensional (3d) microstructures with feature sizes of less than $1 \mu \mathrm{m}$ and allows to create $3 \mathrm{~d}$ organic microlasers such as pyramids with high quality and low surface roughness [2]. Apart from $3 \mathrm{~d}$ bulk resonators it is also possible to fabricate thin, curved waveguides to investigate how light propagates along a curved surface. We investigate the consequences of such a non-Euclidean geometry on the properties of a microlaser.

We fabricate organic microlasers in the shape of a Möbius strip [see Fig. 1(a)] made of a photoresist doped with a laser dye [3]. The Möbius strip is of particular interest since it exhibits topological properties impossible in two dimensions: it has only one surface and one boundary. We investigate how the topology and non-Euclidean geometry of the Möbius strip influences the structure of the lasing modes. In particular, the formation of whispering gallery modes propagating along the boundary as commonly observed for conventional ring resonators is impossible for the Möbius strip since its boundary features both concave and convex parts.

The spectrum of a Möbius microlaser [Fig. 1(b)] is dominated by a series of equidistant peaks, and its Fourier transform [inset of Fig. 1(b)] reveals the optical length of the underlying periodic ray trajectories. The classical ray trajectories in a resonator are determined by Fermat's principle, and on a curved surface, the shortest path between two points is a geodesic. We hence surmise that the lasing modes of a Möbius microlaser are localized on periodic geodesics.

We calculate the modes of a Möbius strip resonator with FDTD simulations and its periodic geodesics with a ray tracing code based on Fermat's principle. Figure 1(c) shows the intensity distribution of a low-loss resonant mode, and its structure is in good agreement with a periodic geodesic with 5 reflections at the boundary. The optical length of this as well as a few other periodic geodesics agrees well with the optical length obtained from the measured lasing spectrum. In conclusion, the experiments and simulations strongly indicate that the lasing modes of the Möbius microlaser are localized on periodic geodesics. These results open the way to further investigations of non-Euclidean photonic devices which can provide new ways of confining and manipulating light in optical microstructures.
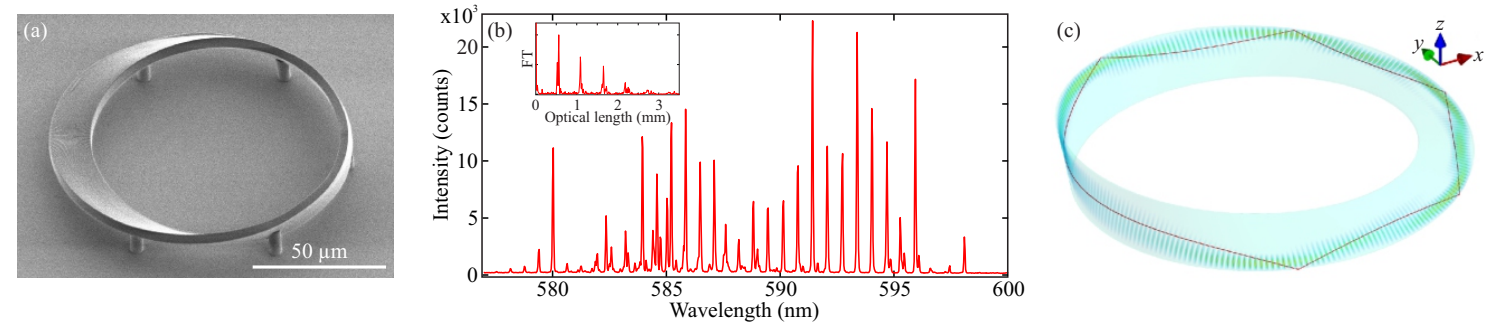

Fig. 1 (a) SEM image of Möbius strip microlaser with radius $50 \mu \mathrm{m}$. (b) Measured lasing spectrum of a $1 \mu \mathrm{m}$ thick Möbius microlaser. Inset: Fourier transform of the laser spectrum. The first peak at about $530 \mu \mathrm{m}$ indicates the length of the underlying trajectories. (c) FDTD calculation of a low-loss resonant mode of a Möbius strip with radius $10 \mu \mathrm{m}$. The corresponding periodic geodesic with 5 vertices is indicated by the red line.

\section{References}

[1] J. Fischer and M. Wegener, “Three-dimensional optical laser lithography beyond the diffraction limit,” Laser \& Phot. Rev. 7, 22 (2013). [2] M. A. Guidry, Y. Song, C. Lafargue, R. Sobczyk, D. Decanin, S. Bittner, B. Dietz, L. Huang, J. Zyss, A. Grigis, and M. Lebental, “Threedimensional micro-billiard lasers: The square pyramid," Europhys. Lett. 126, 64004 (2019).

[3] Y. Song, Y. Monceaux, S. Bittner, K. Chao, H. M. Reynoso de la Cruz, C. Lafargue, D. Decanini, B. Dietz, J. Zyss, A. Grigis, X. Checoury, and M. Lebental, "Möbius strip microlasers: a testbed for non-Euclidian photonics," arXiv:2011.12088 (2020). 